\title{
Immune-Related lncRNA Risk Signatures Predict Survival of IDH Wild-Type and MGMT Promoter Unmethylated Glioblastoma
}

\author{
Xiaozhi $\mathrm{Li}^{1}$ and Yutong Meng $\mathbb{D}^{2}$ \\ ${ }^{1}$ Department of Neurosurgery, Shengjing Hospital of China Medical University, Shenyang, China \\ ${ }^{2}$ Department of Stomatology, Shengjing Hospital of China Medical University, Shenyang, China
}

Correspondence should be addressed to Yutong Meng; mengyt@vip.163.com

Received 9 April 2020; Revised 2 June 2020; Accepted 24 June 2020; Published 12 August 2020

Academic Editor: David A. McClellan

Copyright ( 92020 Xiaozhi Li and Yutong Meng. This is an open access article distributed under the Creative Commons Attribution License, which permits unrestricted use, distribution, and reproduction in any medium, provided the original work is properly cited.

\begin{abstract}
Introduction. Glioblastoma is the most malignant grade of glioma, and it is also the most common primary tumor in the brain. Immunotherapy is a kind of precise tumor treatment. However, there are limited studies about immune-related lncRNA. This study is aimed at analyzing immune-related lncRNAs in glioblastoma and screening out prognostic factors, providing new potential targets for glioblastoma immunology research. Material and Methods. Gene expression data and clinical data of IDH wild-type with MGMT promoter unmethylated glioblastoma were acquired from the TCGA and CGGA databases. Immunerelated lncRNAs were identified with the help of data from the InnateDB database. Immune prognostic factors were recognized by Cox regression analysis. GSEA analysis pursued their potential functions. Results. We found 318 immune-related lncRNAs. Among them, there were 137 immune-related lncRNAs that were upregulated and 181 that were downregulated. 15 prognostic lncRNAs were identified by Cox regression, and a total of 6 molecules were included in the following risk scoring model. GSEA showed that these lncRNAs participated in functions such as protein digestion and absorption and the PPAR signaling pathway. Conclusion. There are limited studies about immune regulation mechanisms of lncRNA in IDH wild-type with MGMT promoter unmethylated glioblastoma. The identified immune-related lncRNAs in glioblastoma might contribute new targets and research directions for immunological molecular studies of glioblastoma.
\end{abstract}

\section{Introduction}

Glioblastoma is the most malignant grade of glioma, and it is also the most common primary tumor in the brain. Despite the existence of neurosurgery, radiotherapy, chemotherapy, and other treatments, the median survival of glioblastoma patients is about 12 to 15 months $[1,2]$. Moreover, the 5year survival rate of glioblastoma is no more than $10 \%[3,4]$. Immunotherapy is a kind of precise tumor treatment, and it is one of the future development directions of cancer treatment. The discovery and application of immunological checkpoints, including PD-1 and CTLA4, provide new directions for the treatment of tumors [5-7]. However, although the anti-PD-1 drug pembrolizumab produced excellent antitumor effects and tolerance in the treatment of melanoma [8], studies have shown that the effect of this drug is far from satisfactory in the treatment of glioblastoma [9-11]. In addition, the immunological mechanisms of glioblastoma are not fully understood. In 2016, WHO conducted a molecular classification for glioma, pointing out the heterogeneity of glioma in various molecular states including IDH mutation status and MGMT promoter methylation status. Meanwhile, the molecular basis and prognosis of glioma patients with different IDH mutation status and MGMT promoter methylation status are significantly different $[12,13]$.

Long-chain noncoding RNAs (lncRNAs) take part in varieties of biological processes such as cell growth, differentiation, posttranscriptional regulation, inflammatory pathology, epigenetic regulation, and subcellular transport [14].

In recent years, some studies on lncRNA related to tumor immunity are being carried out [15-17]. The purpose of this study is to analyze immune-related lncRNA molecules of 
IDH wild-type and MGMT promoter unmethylated glioblastoma and screen out prognostic factors, providing new potential targets for glioblastoma immunology research.

\section{Material and Methods}

2.1. Data Source. Gene expression data and clinical data of IDH wild-type and MGMT promoter unmethylated glioblastoma were obtained from the public CGGA (http://www .cgga.org.cn/) and TCGA (https://cancergenome. https://nih .gov/) databases. CGGA contained two glioma data sets, namely, mRNAseq_325 and mRNAseq_693. TCGA and CGGA gene expression data were corrected using the "limma" package of $\mathrm{R}$ software. Because TCGA data contained gene expression of normal brain tissue, this study used TCGA data for differentially expressed gene screening, TCGA and CGGA (mRNAseq_325) data to construct a risk scoring model, and CGGA (mRNAseq_693) for model verification. Differentially expressed lncRNAs of the TCGA database were identified by the "edgeR" package of $\mathrm{R}$ software. $|\log 2 \mathrm{FC}|>1$ and false discovery rate $<0.05$ were considered statistically different.

2.2. Immune-Related IncRNA Recognition. The list of immunomodulatory-related genes was obtained from the InnateDB website (https://www.innatedb.com). Additionally, when $P<0.05$ and the correlation coefficient $>0.4$, the correlation between RNAs was considered significant.

2.3. Cox Regression and Kaplan-Meier Analysis. Differentially expressed immune-related lncRNAs and clinical data were merged. Univariate Cox regression analysis was applied to find the prognostic ones. Furthermore, the risk score was established by stepwise multivariate Cox regression analysis. The Kaplan-Meier survival curve in high-risk and low-risk groups of glioblastoma was constructed based on the median risk score. The area under the curve (AUC) of the receiver operating characteristic (ROC) curve was applied to assess the clinical prognosis ability of the risk scoring model for glioblastoma patients.

2.4. Gene Set Enrichment Analysis (GSEA). The R software "clusterProfiler" package was used to perform Gene Ontology (GO) enrichment analysis and Kyoto Encyclopedia of Genes and Genomes (KEGG) enrichment analysis between genes in low-risk and high-risk cohorts in order to pursue their possible functions.

2.5. Statistical Analysis. All statistical analyses in this study were performed with $\mathrm{R}$ software 4.0.0. Comparison between groups for abnormal distributed variables was performed by Mann-Whitney $U$ test. Correlation between RNAs was assessed by Pearson correlation test.

\section{Results}

3.1. Differentially Expressed IncRNAs in IDH Wild-Type with MGMT Promoter Unmethylated Glioblastoma. A total of 5 normal brain tissue expression samples and 66 IDH wildtype with MGMT promoter unmethylated glioblastoma samples were included in the TCGA database. The diagnostic age range of glioma patients was 24-89 years old. At the end point of last follow-up, 23 patients survived and 43 died. By the analysis of the "edgeR" package, 318 differentially expressed lncRNAs were identified. There were 137 upregulated and 181 downregulated lncRNAs.

A list of immune-associated genes was acquired from the InnateDB website. A total of 1697 immunomodulatoryrelated genes were extracted. Interestingly, the 318 differentially expressed lncRNAs previously found were all immunerelated genes according to the correlation coefficients between molecules. The corresponding heat map and volcano plot are displayed in Figure 1.

3.2. Univariate and Multivariate Cox Regression. Using TCGA and CGGA (mRNAseq_325) as the training cohort, 15 immune-related prognostic lncRNAs were identified by univariate Cox regression, including LINC00461, LINC00511, CPB2-AS1, AC092171.2, LINC00665, ARHGAP31-AS1, AC006449.1, TRAPPC12-AS1, AC144831.1, TRAM2-AS1, LINC00460, PRRT3-AS1, CACNA1C-AS1, RNF219-AS1, and DNAH10OS. Then, the prognostic model was built by stepwise multivariate Cox regression. A total of 6 lncRNAs were included in the risk scoring model: risk score $=-0.344$ $*$ expression $_{\mathrm{CPB} 2-\mathrm{AS} 1}+0.520 *$ expression $_{\mathrm{AC} 092171.2}-0.341$ $*$ expression $_{\mathrm{LINC} 00665}+0.166 *$ expression $_{\mathrm{LINC} 00460}+0.202 *$ expression $_{\text {PRRT3-AS1 }}+0.298 *$ expression $_{\text {DNAH10OS. }}$ Among them, 2 lncRNAs (CPB2-AS1 and AC092171.2) were independent prognostic factors for IDH wild-type with MGMT promoter unmethylated glioblastoma. The results of the Cox regression are displayed in Table 1 . The heat map of all molecules involved in the risk score is shown in Figure 2(a). Besides, the relationship between risk scores and overall survival is shown in Figure 2(b).

In order to verify the ability of predicting overall survival of glioma patients, glioblastoma samples were divided into low-risk and high-risk groups based on the median value of the risk score. Through the Kaplan-Meier survival curves, overall survival of low-risk patients was much higher than that of their high-risk counterparts (training cohort $P<0.001$; validation cohort $P=0.035$ ), displayed in Figure 3(a). In addition, ROC curves of the training group and the verification group are displayed in Figure 3(b). The AUC values of 1year and 3-year ROC curves were 0.741 and 0.869 , respectively, in the training cohort, while in the verification group, the AUC values of the 1-year and 3-year ROC curves were 0.554 and 0.728 , respectively. The ROC curves showed that the risk score had a good predictive ability for the prognosis of glioblastoma.

In addition, the expression of CPB2-AS1, AC092171.2, LINC00665 and LINC00460, PRRT3-AS1, and DNAH10OS in each subtype of glioblastoma is shown in Figure 4.

3.3. GSEA. We performed the functional enrichment analysis on the differentially expressed lncRNAs (Figure 5). The GO enrichment analysis suggested that the differential expressed lncRNAs were enriched in items such as extracellular matrix organization, collagen-containing extracellular matrix, and extracellular matrix structure constituent. KEGG analysis showed that these lncRNAs were involved in pathways such 


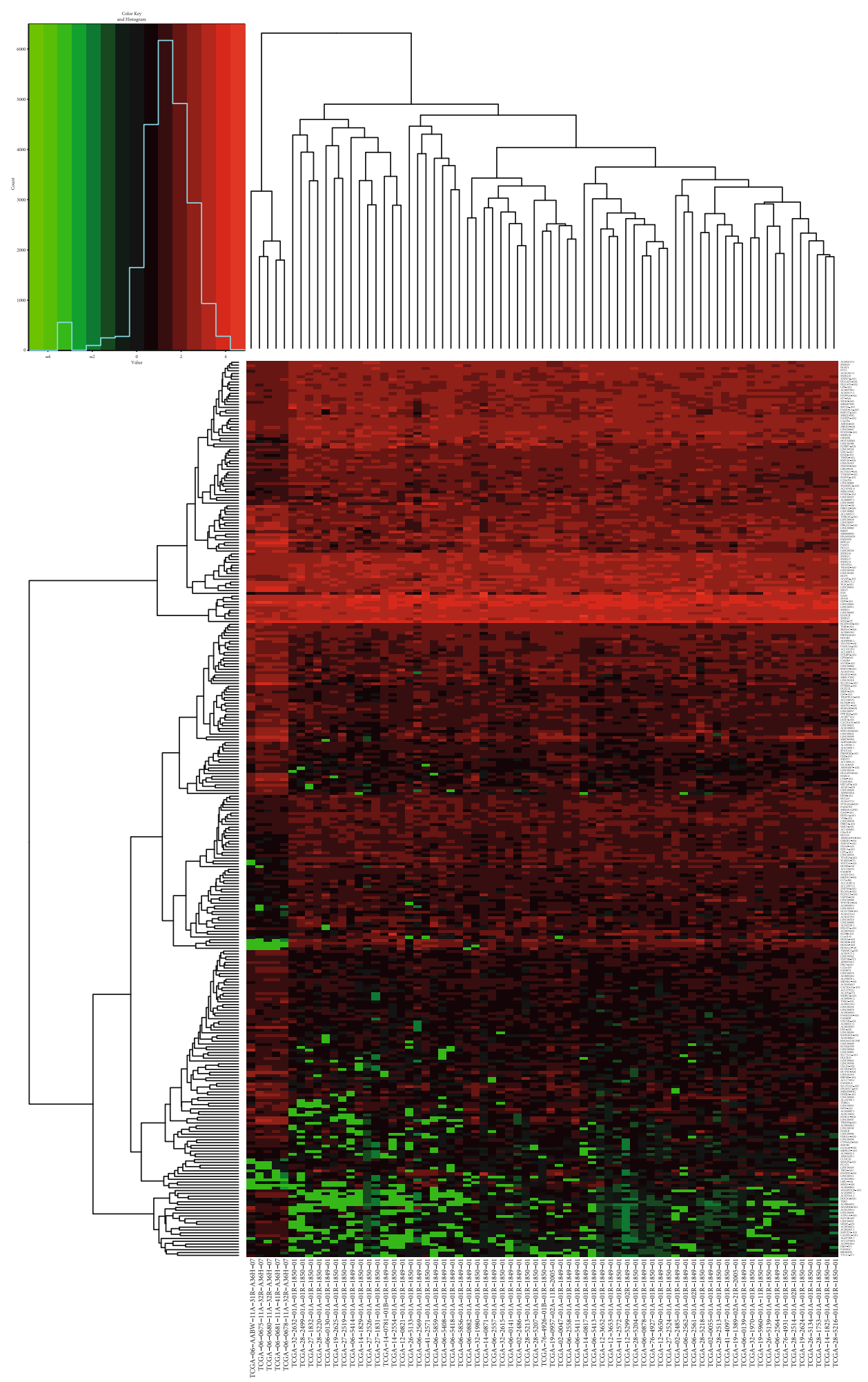

(a)

FIgUre 1: Continued. 


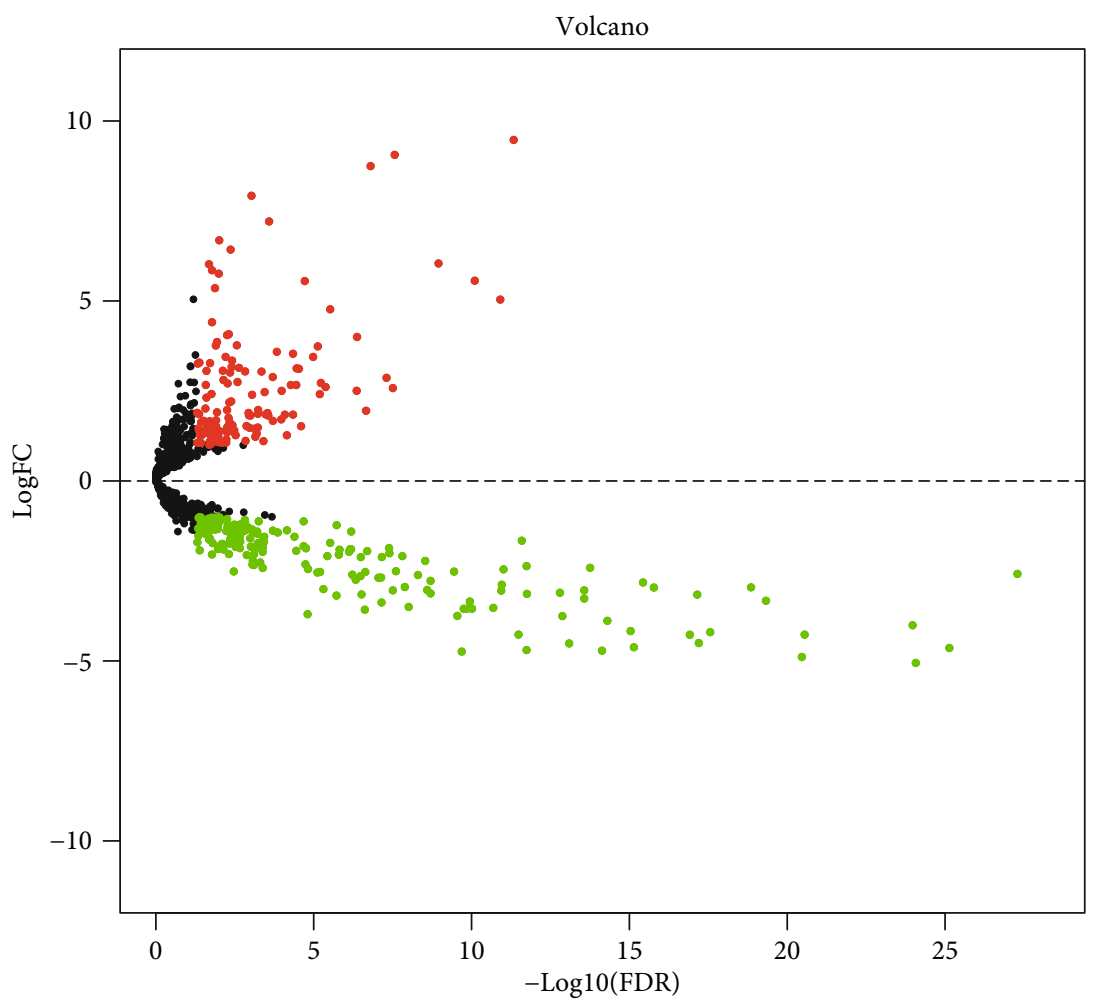

(b)

Figure 1: Differential expression immune-related lncRNAs. (a) Heat map of immune-related lncRNAs. Red blocks represent upregulation whereas green blocks represent downregulation. (b) Volcano plot of immune-related lncRNAs. Red points represent upregulation whereas green points represent downregulation.

TABLE 1: The Cox regression results of immune-related lncRNAs. Univariate analysis and multivariate cox regression of immune-associated lncRNAs.

\begin{tabular}{|c|c|c|c|c|}
\hline \multirow{2}{*}{$\operatorname{lncRNA}$} & \multicolumn{2}{|c|}{ Univariate analysis } & \multicolumn{2}{|c|}{ Multivariate analysis } \\
\hline & HR (95\% CI) & $P$ & HR (95\% CI) & $P$ \\
\hline CPB2-AS1 & $0.599(0.459-0.781)$ & 0.000 & $0.709(0.547-0.918)$ & 0.009 \\
\hline AC092171.2 & $1.587(1.076-2.340)$ & 0.020 & $1.681(1.074-2.634)$ & 0.023 \\
\hline LINC00665 & $0.653(0.462-0.922)$ & 0.016 & $0.711(0.489-1.033)$ & 0.073 \\
\hline LINC00460 & $1.279(1.088-1.504)$ & 0.003 & $1.180(0.987-1.411)$ & 0.060 \\
\hline PRRT3-AS1 & $1.241(1.006-1.531)$ & 0.044 & $1.224(0.954-1.569)$ & 0.112 \\
\hline DNAH10OS & $1.477(1.102-1.980)$ & 0.009 & $1.347(0.966-1.876)$ & 0.079 \\
\hline
\end{tabular}

as protein digestion and absorption and PPAR signaling pathway.

\section{Discussion}

The treatment of glioblastoma is challenging. Immune checkpoints can modulate the magnitude and quality of $\mathrm{T}$ cell responses, and tumors use these mechanisms to inactivate $\mathrm{T}$ cells in an immunosuppressive microenvironment [18]. The production of the immunosuppressive microenvironment (TME) makes immunotherapy effects in patients with glioblastoma unsatisfactory [19]. Checkpoint inhibitors such as anti-PD-1, anti-PDL1, and anti-CTLA4 have been used in many tumors. In a mouse model of glioblastoma, anti-PD-1 therapy combined with TMZ can eradicate glioblastoma
[20]. However, single checkpoint inhibitors have little effect on the treatment of most GBM patients [21]. This may be due to the specificity of glioblastoma immunization and the heterogeneity of glioblastoma itself, so more in-depth studies are needed to identify new and effective checkpoints. Glioblastoma immune-microenvironment presents an antitumor immune response. Though little is known about immunosuppression, regulation of the immune system is crucial for the treatment of glioblastoma [22].

In this study, differentially expressed lncRNAs of the IDH wild-type with MGMT promoter unmethylated glioblastoma were analyzed, and Cox regression was used to construct a prognostic model for glioma patients. KaplanMeier curves and ROC curves suggested that the risk scoring model based on the 6 lncRNAs (CPB2-AS1, AC092171.2, 


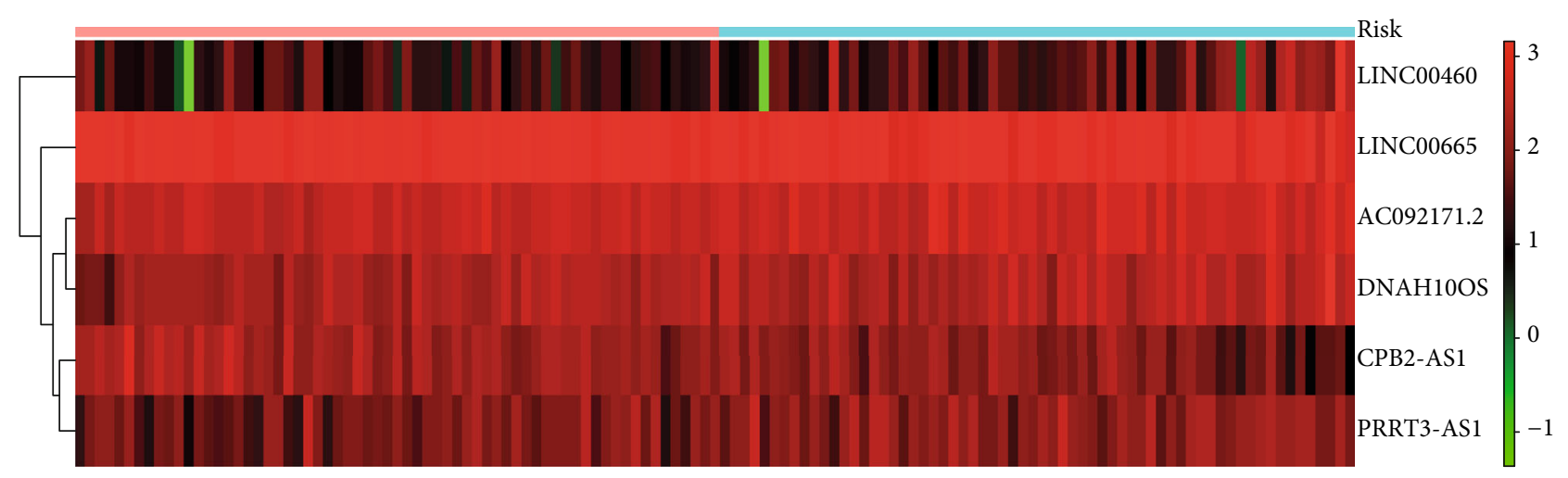

Risk

High

Low

(a)
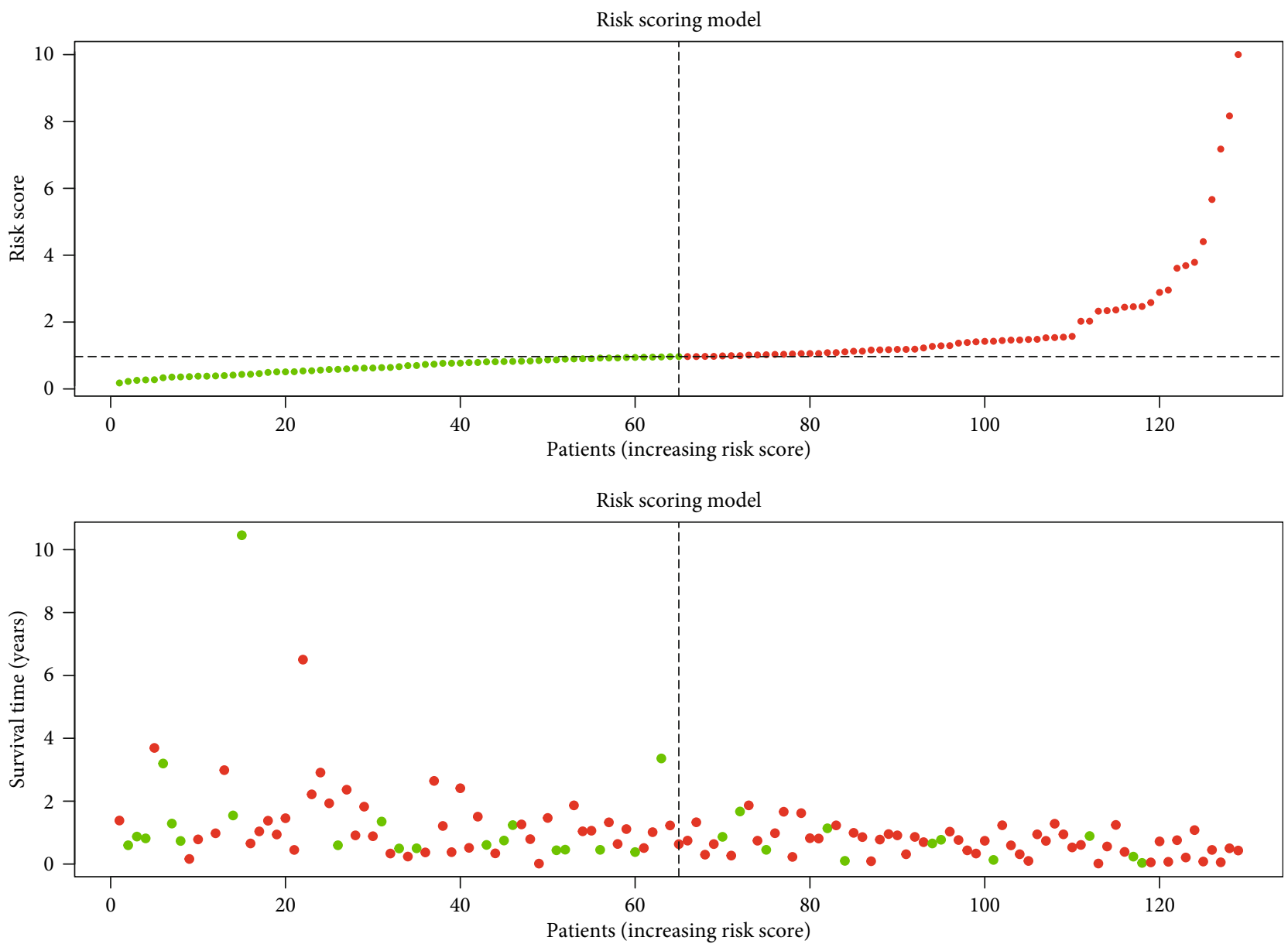

(b)

FIGURE 2: Prognostic immune lncRNAs. (a) Heat map of prognostic immune lncRNAs. (b) The relationship between risk scores and overall survival.

LINC00665 and LINC00460, PRRT3-AS1, and DNAH10OS) had a strong predictive ability for glioblastoma patients.

There have been some lncRNA analyses of glioma. For example, Li et al. has applied immune lncRNA analysis for pancancer characterization based on the TCGA database [23]. Moreover, we have also previously conducted studies on a lncRNA immune study of low-grade gliomas based on the TCGA database [24]. Zhou et al. analyzed all the IncRNAs of glioblastomas based on the TCGA database 


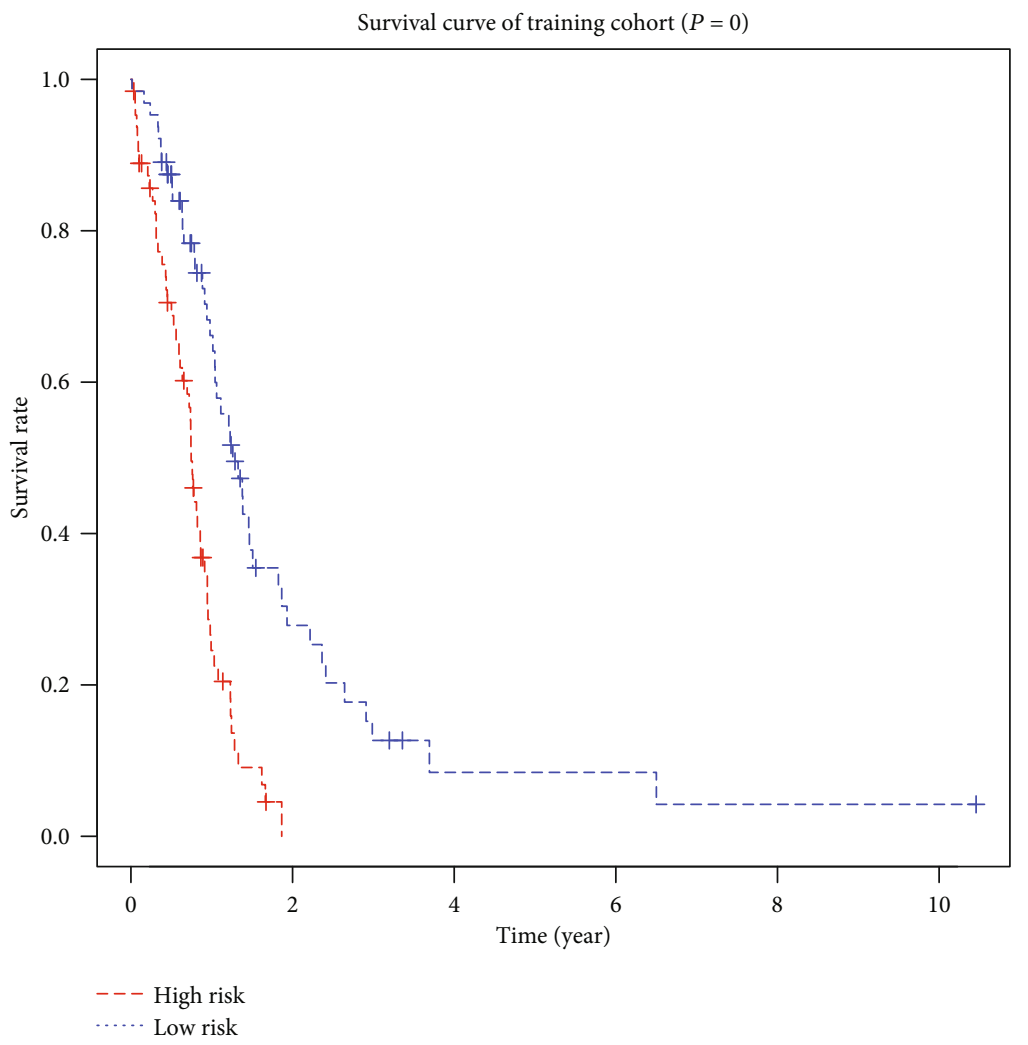

(a)

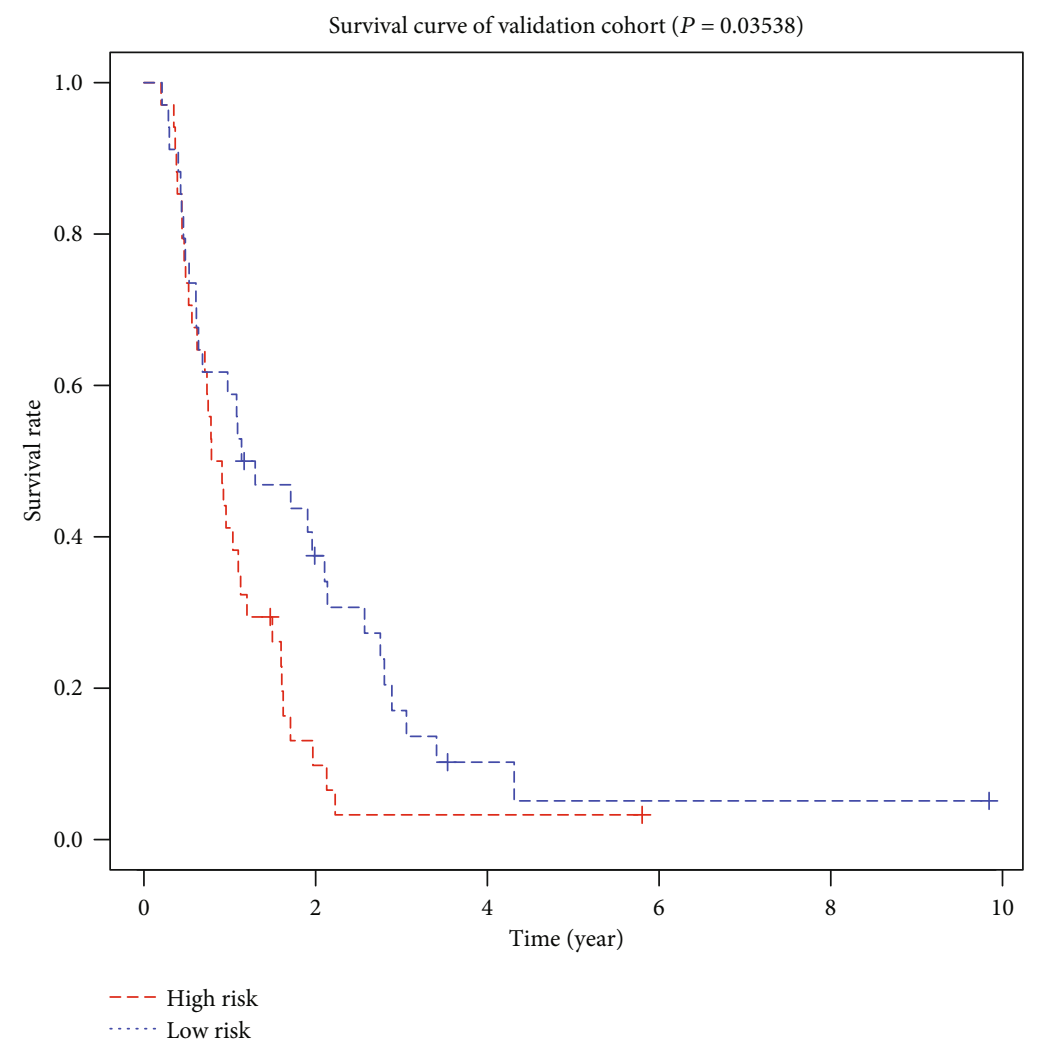

(b)

Figure 3: Continued. 


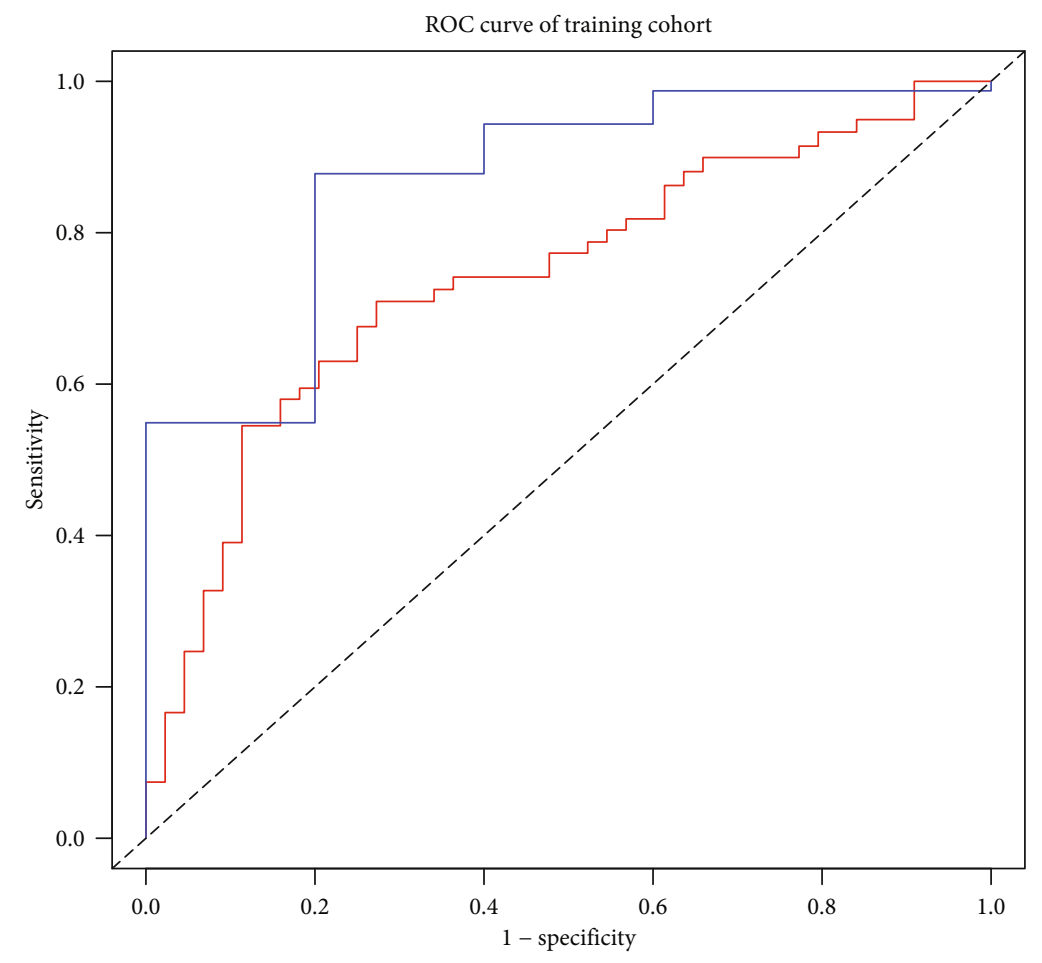

- AUC of 1-year survival: 0.741 — AUC of 3-year survival: 0.869

(c)

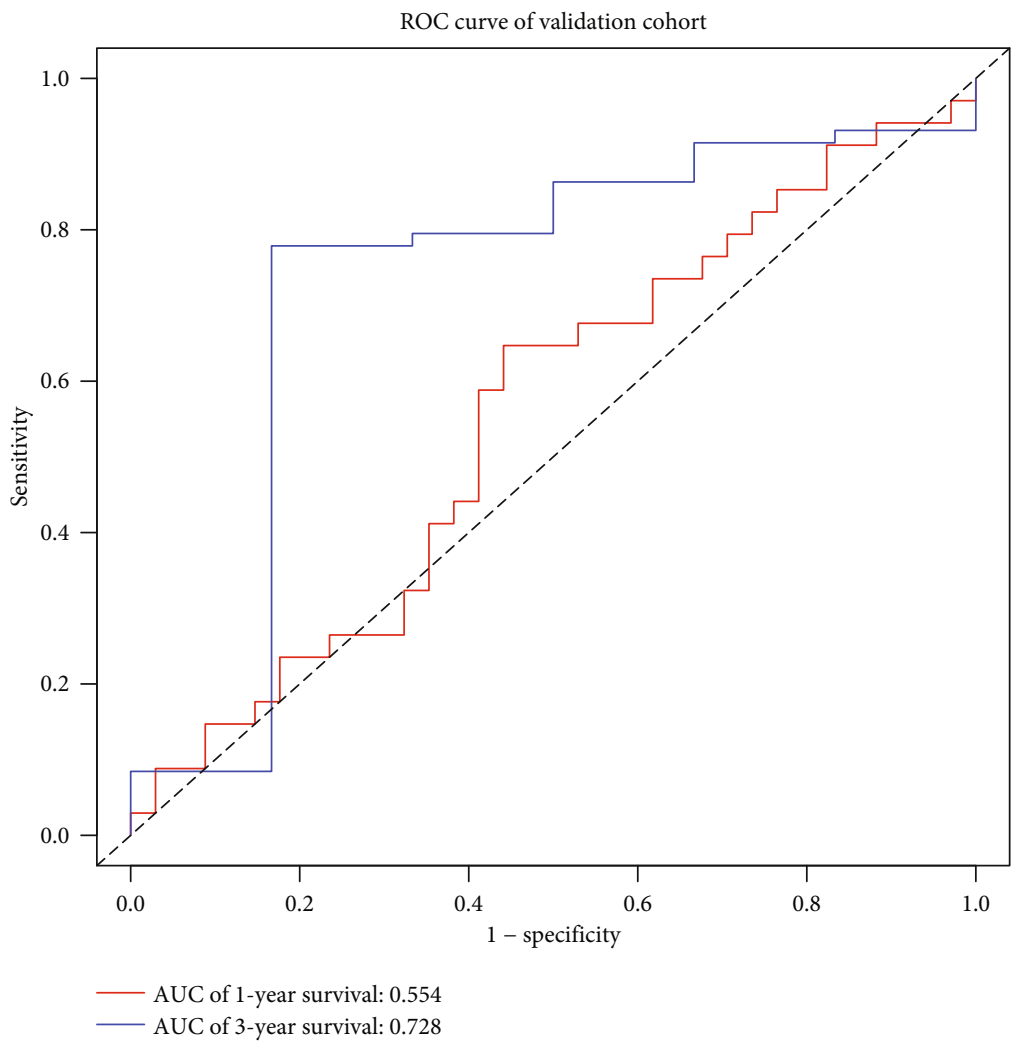

(d)

FIGURE 3: Testing of the construction of the risk scoring model. (a) Kaplan-Meier survival curve of the training cohort. (b) Kaplan-Meier survival curve of the validation cohort. (c) The ROC curve of the training cohort. (d) The ROC curve of the validation cohort. 

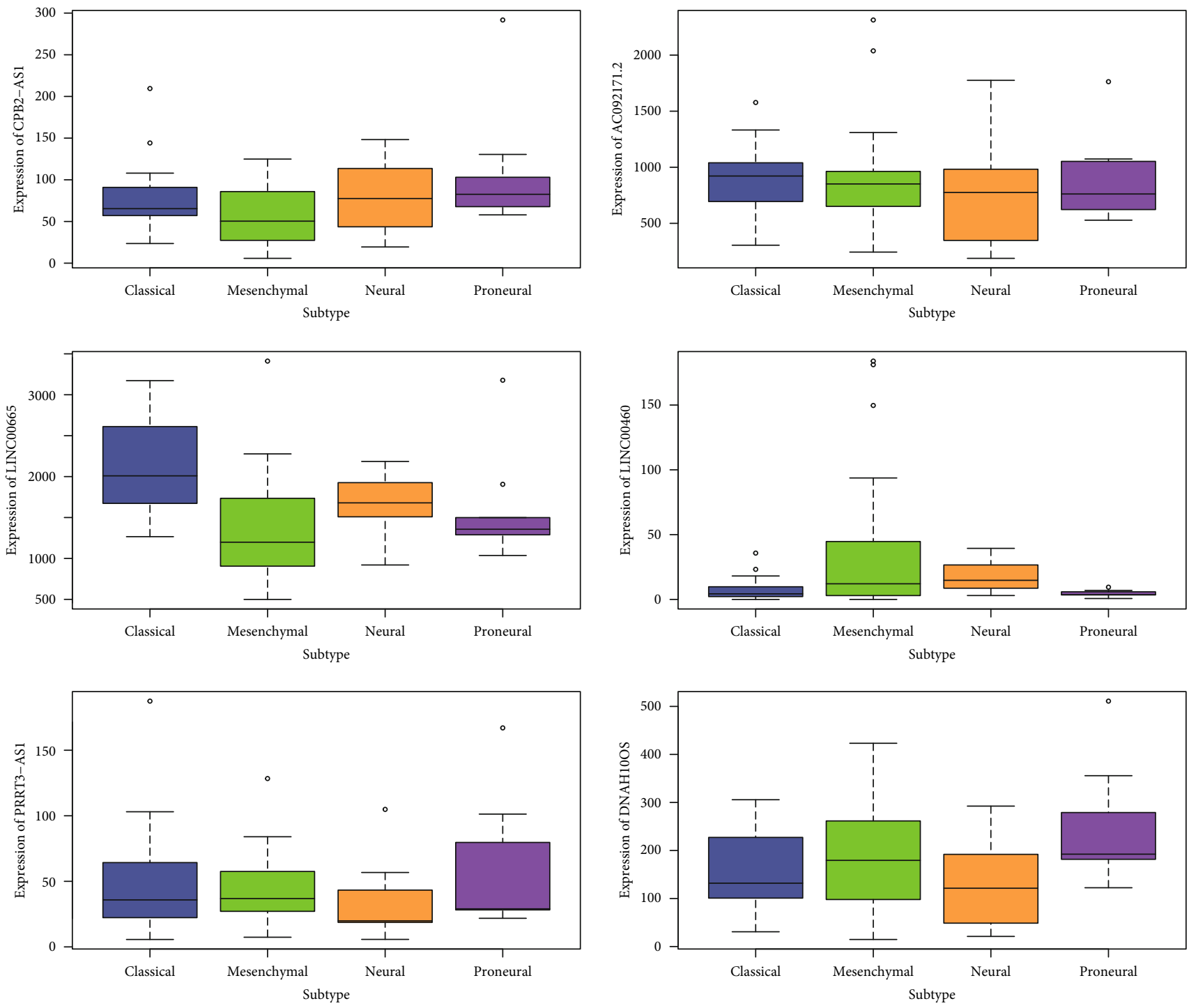

Figure 4: 6 lncRNA (CPB2-AS1, AC092171.2, LINC00665 and LINC00460, PRRT3-AS1, and DNAH10OS) expression in subtypes of glioblastoma.

[25]. This study, due to the strong difference in molecular bases and prognosis of glioblastoma patients with different IDH mutation with MGMT promoter methylation status, conducted a multidatabase analysis (TCGA and CGGA databases) to immune-related lncRNA analysis of the IDH wildtype and MGMT promoter unmethylated glioblastoma, to provide targets for immunological studies of glioblastoma.

Through the correlation analysis, 6 immune-related lncRNAs were identified, some of which have been reported in the previous studies. For example, LINC00665 is downregulated in glioma and mediates STAU1-mediated MTF1 and YY2 stability, affecting malignant biological behavior of glioma [26]. Besides, LINC00460 is upregulated in glioma. LINC00460 can promote the proliferation, migration, and invasion of glioma cells by negatively regulating miR-320a [27]. In prostate cancer, PRRT3-AS1 silencing can block the mTOR signaling pathway, promote tumor cell autophagy, and inhibit tumor cell proliferation [28]. However,
PRRT3-AS1 has not been reported involving immune regulation of glioma.

There are limitations in the study. First of all, this study is a retrospective study based on high-throughput sequencing data and requires the verification of many further basic experiments. Secondly, this study focuses on the IDH wild-type with MGMT promoter unmethylated glioblastoma. A more detailed comparison of molecular subtypes of glioblastoma may require larger sequencing data to obtain better results.

\section{Conclusion}

This study identified a total of 318 immune-related lncRNAs in IDH wild-type with MGMT promoter unmethylated glioblastoma. Univariate Cox regression reveals 15 prognosisrelated lncRNAs. Six lncRNAs were considered to be independent prognostic risk factors for glioblastoma. GO and KEGG analyses suggested that the lncRNAs may involve 


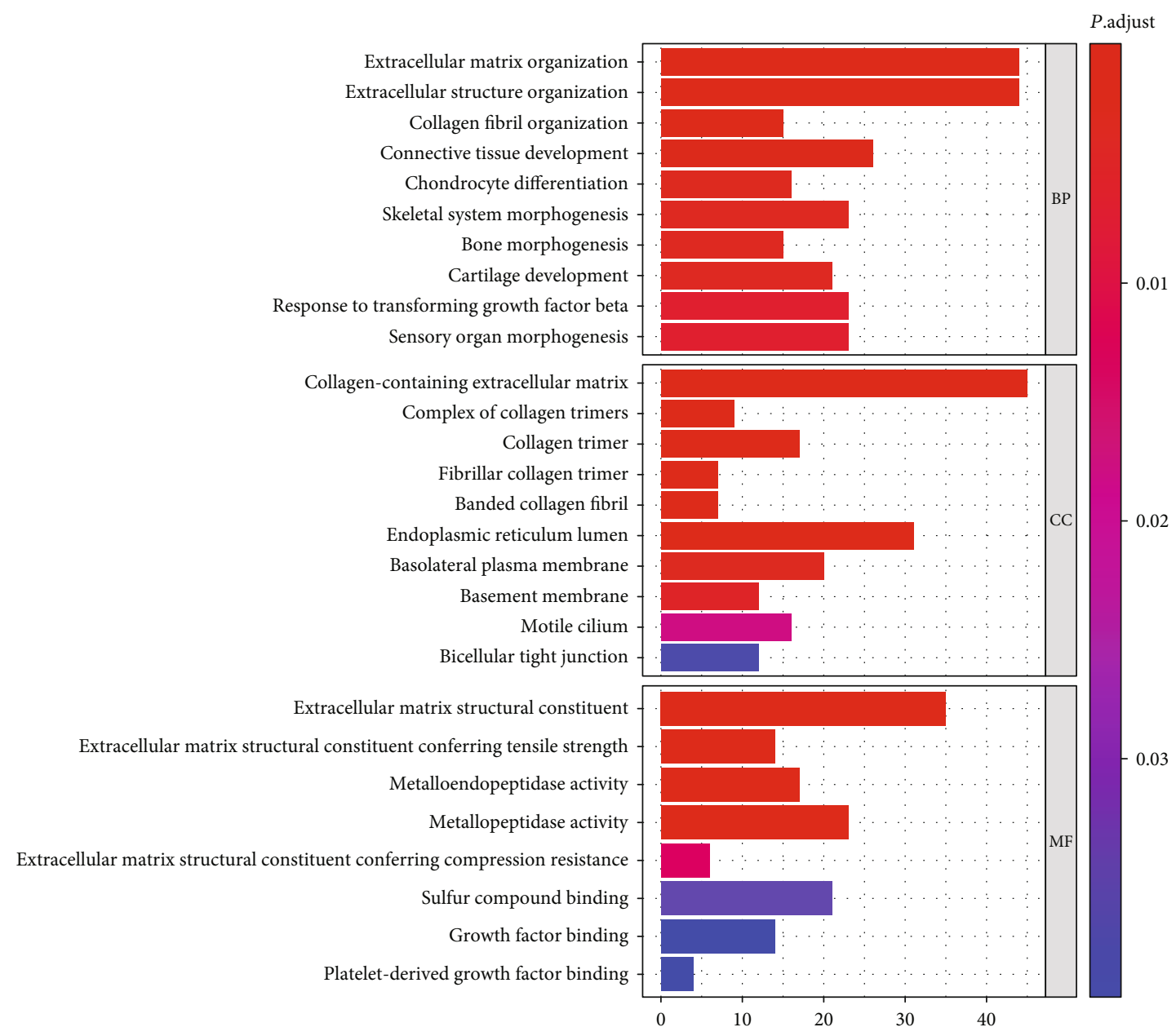

(a)

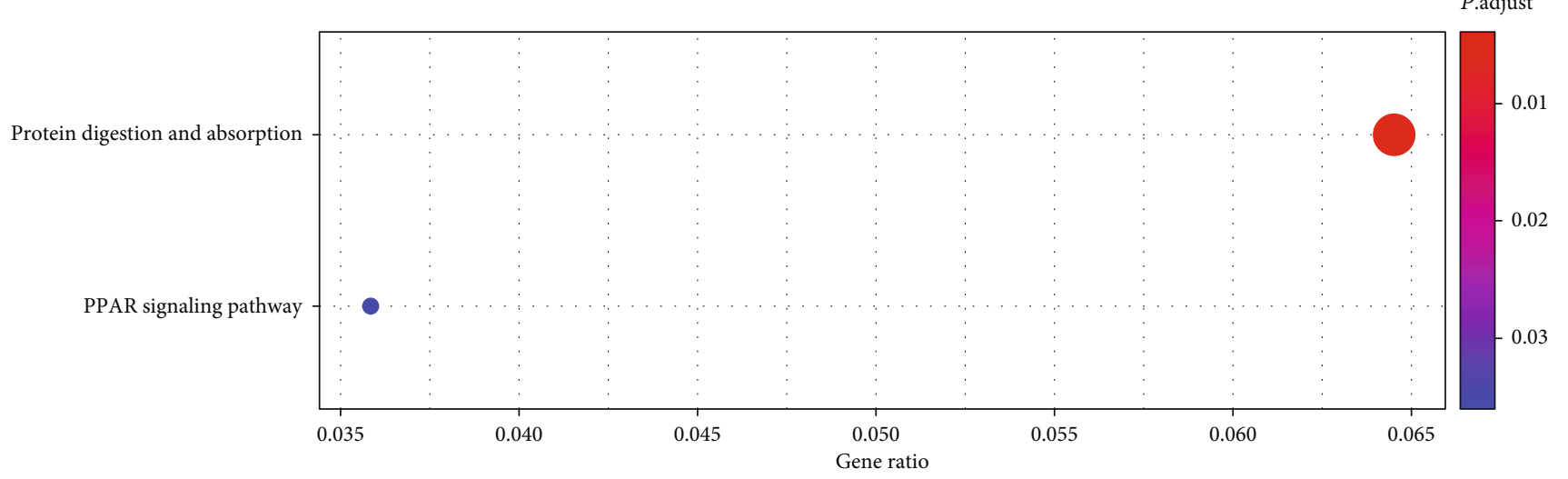

Count

10
12
14

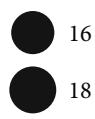

(b)

Figure 5: GSEA: (a) GO analysis results; (b) KEGG analysis results.

signaling pathways such as extracellular matrix organization, collagen-containing extracellular matrix, extracellular matrix structure constituent, protein digestion and absorption, and
PPAR signaling pathway. This study is aimed at contributing new targets and research directions for immunological molecular studies of glioblastoma. 


\section{Data Availability}

The data in this study was available from the corresponding author on reasonable request.

\section{Conflicts of Interest}

The authors declare that they have no conflicts of interest.

\section{Authors' Contributions}

YM contributed to the experiment design and manuscript draft. XL contributed to the manuscript draft and data analysis. All authors have read and approved the final manuscript.

\section{Acknowledgments}

The study was supported by the 345 Talent Project.

\section{References}

[1] A. Roos, Z. Ding, J. C. Loftus, and N. L. Tran, "Molecular and microenvironmental determinants of glioma stem-like cell survival and invasion," Frontiers in Oncology, vol. 7, p. 120, 2017.

[2] R. Stupp, M. E. Hegi, W. P. Mason et al., "Effects of radiotherapy with concomitant and adjuvant temozolomide versus radiotherapy alone on survival in glioblastoma in a randomised phase III study: 5-year analysis of the EORTC-NCIC trial," The Lancet Oncology, vol. 10, no. 5, pp. 459-466, 2009.

[3] R. Batash, N. Asna, P. Schaffer, N. Francis, and M. Schaffer, "Glioblastoma multiforme, diagnosis and treatment; recent literature review," Current Medicinal Chemistry, vol. 24, no. 27, pp. 3002-3009, 2017.

[4] R. Stupp, W. P. Mason, M. J. van den Bent et al., "Radiotherapy plus concomitant and adjuvant temozolomide for glioblastoma," The New England Journal of Medicine, vol. 352, no. 10, pp. 987-996, 2005.

[5] M. Caccese, S. Indraccolo, V. Zagonel, and G. Lombardi, "PD1/PD-L1 immune-checkpoint inhibitors in glioblastoma: a concise review," Critical Reviews in Oncology/Hematology, vol. 135, pp. 128-134, 2019.

[6] R. Q. Chen, F. Liu, X. Y. Qiu, and X. Q. Chen, "The prognostic and therapeutic value of PD-L1 in glioma," Frontiers in Pharmacology, vol. 9, 2019.

[7] Q. Ma, W. Long, C. Xing et al., "Cancer stem cells and immunosuppressive microenvironment in glioma," Frontiers in Immunology, vol. 9, 2018.

[8] O. Hamid, C. Robert, A. Daud et al., "Five-year survival outcomes for patients with advanced melanoma treated with pembrolizumab in KEYNOTE-001," Annals of Oncology, vol. 30, no. 4, pp. 582-588, 2019.

[9] D. T. Blumenthal, M. Yalon, G. W. Vainer et al., "Pembrolizumab: first experience with recurrent primary central nervous system (CNS) tumors," Journal of Neuro-Oncology, vol. 129, no. 3, pp. 453-460, 2016.

[10] S. C. Kurz, L. P. Cabrera, D. Hastie et al., "PD-1 inhibition has only limited clinical benefit in patients with recurrent highgrade glioma," Neurology, vol. 91, no. 14, pp. e1355-e1359, 2018.
[11] S. N. Reiss, P. Yerram, L. Modelevsky, and C. Grommes, "Retrospective review of safety and efficacy of programmed cell death-1 inhibitors in refractory high grade gliomas," Journal for Immunotherapy of Cancer, vol. 5, no. 1, 2017.

[12] A. Pellerino, F. Franchino, R. Soffietti, and R. Rudà, "Overview on current treatment standards in high-grade gliomas," The Quarterly Journal of Nuclear Medicine and Molecular Imaging, vol. 62, no. 3, pp. 225-238, 2018.

[13] Y. Sonoda, "Clinical impact of revisions to the WHO classification of diffuse gliomas and associated future problems," International Journal of Clinical Oncology, vol. 25, no. 6, pp. 10041009, 2020.

[14] R. Rynkeviciene, J. Simiene, E. Strainiene et al., "Non-coding RNAs in glioma," Cancers, vol. 11, no. 1, 2019.

[15] J. Sun, Z. Zhang, S. Bao et al., "Identification of tumor immune infiltration-associated lncRNAs for improving prognosis and immunotherapy response of patients with non-small cell lung cancer," Journal for ImmunoTherapy of Cancer, vol. 8, no. 1, 2020.

[16] M. Zhou, Z. Zhang, S. Bao et al., "Computational recognition of lncRNA signature of tumor-infiltrating B lymphocytes with potential implications in prognosis and immunotherapy of bladder cancer," Briefings in Bioinformatics, 2020.

[17] M. Zhou, H. Zhao, W. Xu, S. Bao, L. Cheng, and J. Sun, "Discovery and validation of immune-associated long non-coding RNA biomarkers associated with clinically molecular subtype and prognosis in diffuse large B cell lymphoma," Molecular Cancer, vol. 16, no. 1, 2017.

[18] M. Preusser, M. Lim, D. A. Hafler, D. A. Reardon, and J. H. Sampson, "Prospects of immune checkpoint modulators in the treatment of glioblastoma," Nature Reviews Neurology, vol. 11, no. 9, pp. 504-514, 2015.

[19] W. Tomaszewski, L. Sanchez-Perez, T. F. Gajewski, and J. H. Sampson, "Brain tumor microenvironment and host state: implications for immunotherapy," Clinical Cancer Research, vol. 25, no. 14, pp. 4202-4210, 2019.

[20] J. Park, C. G. Kim, J.-K. Shim et al., "Effect of combined antiPD-1 and temozolomide therapy in glioblastoma," Oncoimmunology, vol. 8, no. 1, article e1525243, 2019.

[21] T. McGranahan, K. E. Therkelsen, S. Ahmad, and S. Nagpal, "Current state of immunotherapy for treatment of glioblastoma," Current Treatment Options in Oncology, vol. 20, no. 3, 2019.

[22] S. Luoto, I. Hermelo, E. M. Vuorinen et al., "Computational characterization of suppressive immune microenvironments in glioblastoma," Cancer Research, vol. 78, no. 19, pp. 5574$5585,2018$.

[23] Y. Li, T. Jiang, W. Zhou et al., "Pan-cancer characterization of immune-related lncRNAs identifies potential oncogenic biomarkers," Nature Communications, vol. 11, no. 1, 2020.

[24] X. Li and Y. Meng, "Survival analysis of immune-related lncRNA in low-grade glioma," BMC Cancer, vol. 19, no. 1, 2019.

[25] M. Zhou, Z. Zhang, H. Zhao, S. Bao, L. Cheng, and J. Sun, “An immune-related six-lncRNA signature to improve prognosis prediction of glioblastoma multiforme," Molecular Neurobiology, vol. 55, no. 5, pp. 3684-3697, 2018.

[26] X. Ruan, J. Zheng, X. Liu et al., "IncRNA LINC00665 stabilized by TAF15 impeded the malignant biological behaviors of glioma cells via STAU1-mediated mRNA degradation," Molecular Therapy Nucleic Acids, vol. 20, pp. 823-840, 2020. 
[27] L. Feng, M. Rao, Y. Zhou, Y. Zhang, and Y. Zhu, "Long noncoding RNA 00460 (LINC00460) promotes glioma progression by negatively regulating miR-320a," Journal of Cellular Biochemistry, vol. 120, no. 6, pp. 8801-10863, 2019.

[28] L. Fan, H. Li, and W. Wang, "Long non-coding RNA PRRT3AS1 silencing inhibits prostate cancer cell proliferation and promotes apoptosis and autophagy," Experimental Physiology, vol. 105, no. 5, pp. 793-808, 2020. 